International Journal of Applied Mathematics

Volume 29 No. 1 2016, 1-6

ISSN: 1311-1728 (printed version); ISSN: 1314-8060 (on-line version)

doi: http://dx.doi.org/10.12732/ijam.v29i1.1

\title{
GENERALIZATION OF RIEMANN-HILBERT BOUNDARY \\ VALUE PROBLEM FOR A FIRST ORDER NONLINEAR \\ COMPLEX PARTIAL DIFFERENTIAL EQUATION
}

\author{
N. Taghizadeh ${ }^{1}$, H. Hoseini ${ }^{2} \S$ \\ ${ }^{1,2}$ Department of Mathematics \\ Faculty of Mathematical Sciences \\ University of Guilan \\ P.O. Box 1914, Rasht, IRAN
}

Abstract: In this paper we discuss on the existence and uniqueness solution of the Riemann-Hilbert boundary value problem in the form:

$$
\begin{gathered}
\frac{\partial w}{\partial \bar{z}}=F\left(z, w, \frac{\partial w}{\partial z}\right)+G(z, w, \bar{w}), \quad z \in D, \\
\operatorname{Re}(a+i b) w=g \quad \text { on } \quad \partial D
\end{gathered}
$$

in $C_{1, \alpha}(\bar{D})$, where $a, b$ and $g$ are given Holder continuously differentiable realvalued functions of a real parameter $t$ on $\partial D$. We shall assume that $a^{2}+b^{2}=1$ everywhere on $\partial D$.

AMS Subject Classification: 35D05, 35J55, 35J67

Key Words: boundary value problem, Banach space, fixed point theorem, complex differential equation, holomorphic function

Received: August 15, 2015

(C) 2016 Academic Publications

${ }^{\S}$ Correspondence author 


\section{Introduction}

Suppose that $D$ is a bounded domain belonging to the class $C_{1, \alpha}$ in the complex plane and $F\left(z, w, \frac{\partial w}{\partial z}\right) \in C_{\alpha}(\bar{D}), 0<\alpha<1$, we define the weakly and strongly singular operators $T_{D}$ and $\prod_{D}$ in the form:

$$
\begin{gathered}
T_{D} f(z)=-\frac{1}{\pi} \iint_{D} \frac{1}{\xi-z} f(\xi) d \zeta d \eta, \\
\prod_{D} f(z)=-\frac{1}{\pi} \iint_{D} \frac{1}{(\xi-z)^{2}} f(\xi) d \zeta d \eta,
\end{gathered}
$$

where $\xi=\zeta+i \eta, z=x+i y$, and if $f \in L_{p}(D)$ then $T_{D} f$ is bounded and Holder continuous, [1], so that

$$
\begin{gathered}
\frac{\partial T_{D} f(z)}{\partial \bar{z}}=f(z), \\
\frac{\partial T_{D} f(z)}{\partial z}=\prod_{D} f(z) .
\end{gathered}
$$

Furthermore, we assume that $w \in C_{\alpha}(\bar{D}), 0<\alpha<1$, is an arbitrary solution of:

$$
\frac{\partial w}{\partial \bar{z}}=F\left(z, w, \frac{\partial w}{\partial z}\right)+G(z, w, \bar{w}) .
$$

We define a function $\Phi$ as follows:

$$
\Phi(z)=w(z)-T_{D}\left[F\left(z, w, \frac{\partial w}{\partial z}\right)+G(z, w, \bar{w})\right],
$$

on differentiating $\Phi$ partially with respect to $\bar{z}$ and $z$ respectively, we obtain the following

$$
\left\{\begin{array}{l}
\frac{\partial \Phi}{\partial \bar{z}}=\frac{\partial w}{\partial \bar{z}}-\left[F\left(z, w, \frac{\partial w}{\partial z}\right)+G(z, w, \bar{w})\right] \\
\frac{\partial \Phi}{\partial z}=\frac{\partial w}{\partial z}-\prod_{D}\left[F\left(z, w, \frac{\partial w}{\partial z}\right)+G(z, w, \bar{w})\right]
\end{array} .\right.
$$

Furthermore, since $F\left(z, w, \frac{\partial w}{\partial z}\right) \in C_{\alpha}(\bar{D}), 0<\alpha<1$, the following estimates hold:

$$
\begin{aligned}
\|\Phi\|_{\alpha, D} & \leq\|w\|_{\alpha, D}+\left\|T_{D}(F+G)\right\|_{\alpha, D}, \\
\left\|\frac{\partial \Phi}{\partial z}\right\|_{\alpha, D} & \leq\left\|\frac{\partial w}{\partial z}\right\|_{\alpha, D}+\left\|\prod_{D}(F+G)\right\|_{\alpha, D} .
\end{aligned}
$$


It follows from the first equation (4) and Wely's lemma [6] that $\Phi$ is holomorphic function in $D$, it belong to the class $C_{\alpha}(\bar{D}), 0<\alpha<1$. Moreover, we deduce that, if $w$ is a solution of (1), then $w$ necessarily is of the form

$$
w(z)=\Phi(z)+T_{D}\left[F\left(z, w, \frac{\partial w}{\partial z}\right)+G(z, w, \bar{w})\right],
$$

where $\Phi$ is holomorphic in $D$. We now suppose that $(w, h)$ is a solution of the following system:

$$
\left\{\begin{array}{rl}
w(z) & =\Phi(z)+T_{D}[F(z, w, h)+G(z, w, \bar{w})] \\
h(z) & =\Phi^{\prime}(z)+\prod_{D}[F(z, w, h)+G(z, w, \bar{w})]
\end{array} .\right.
$$

Then $w$ is a solution to the given differential equation(1). On substituting $h=\frac{\partial w}{\partial z}$ in (6) we obtain the following result.

Theorem 1.1. A function $w \in C_{1, \alpha}(\bar{D})$ in the form in (5), is a solution to the partial differential equation (1) if and only if, for a holomorphic function $\Phi \in C_{1, \alpha}(\bar{D}),(w, h)$ is the solution of the system (6).

\section{Existence of a General Solution in $C_{1, \alpha}(D)$}

In order to determine the existence of a solution $w \in C_{1, \alpha}(\bar{D})$, we shall work in the following space $\mathcal{J}_{\alpha}(\bar{D}), 0<\alpha<1$ : we denote by $\mathcal{J}_{\alpha}(\bar{D})$ the set of all pairs $(w, h)$ for which both $w$ and $h$ belong to the space $C_{\alpha}(\bar{D})$. The norm shall be defined as follows:

$$
\|(w, h)\|_{\alpha, D}:=\max \left(\|w\|_{\alpha, D},\|h\|_{\alpha, D}\right),
$$

thus the $\mathcal{J}_{\alpha}(\bar{D})$ becomes a Banach space.

We impose the following assumptions on the complex differential equation (1):

I. The domain $D$ is bounded and belongs to the class $C_{1, \alpha}, 0<\alpha<1$.

II. The right hand side $F(z, w, h)$ is a continuous function of $z \in D, w, h$.

III. The function $F(z, w, h) \in C_{\alpha}(\bar{D})$ if $w, h \in C_{\alpha}(\bar{D})$.

IV. $F(z, w, h)$ satisfies a Lipschitz condition in the metric of Hölder norm:

$$
\begin{gathered}
\|F(z, w, h)-F(z, \tilde{w}, \tilde{h})\|_{\alpha, D} \leq L_{1}\|(w, h)-(\tilde{w}, \tilde{h})\|_{\alpha, D} \\
\| G(z, w, \bar{w})-G\left(z, \tilde{w}, \overline{\tilde{w}}\left\|_{\alpha, D} \leq L_{2}\right\| w-\bar{w} \|_{\alpha, D}\right.
\end{gathered}
$$


The assumption III is satisfied if the following Lipschitz condition is satisfied:

$$
\begin{gathered}
|F(z, w, h)-F(\tilde{z}, \tilde{w}, \tilde{h})| \leq L\left[|z-\tilde{z}|^{\alpha}+\max (|w-\tilde{w}|,|h-\tilde{h}|)\right] \\
z, \tilde{z} \in \bar{D} \quad \text { and }(w, h),(\tilde{w}, \tilde{h}) \in \mathcal{J}_{\alpha}(\bar{D}) .
\end{gathered}
$$

In this case we have

$$
|F(z, w, h)-F(\tilde{z}, \tilde{w}, \tilde{h})| \leq L_{1}[1+\max (H(\alpha, w), H(\alpha, h))]|z-\tilde{z}|^{\alpha},
$$

where

$$
H(\alpha, h)=\sup \frac{|h(z)-h(\tilde{z})|}{|z-\tilde{z}|^{\alpha}}, \quad z, \tilde{z} \in D .
$$

With the aid of the right hand side of (5) we now define an operator $Q$ as follows: For $(w, h) \in \mathcal{J}_{\alpha}(\bar{D})$, let $Q(w, h)=(W, H)$ :

$$
\begin{aligned}
& W(z)=\Phi(z)+T_{D}[F(z, w, h)+G(z, w, \bar{w})], \\
& H(z)=\Phi^{\prime}(z)+\prod_{D}[F(z, w, h)+G(z, w, \bar{w})],
\end{aligned}
$$

where $\Phi$ is a holomorphic function in $D$ and $\Phi \in C_{1, \alpha}(\bar{D})$.

By the properties of the integral operators $T_{D}, \prod_{D}$ in $C_{\alpha}(\bar{D})$, we conclude that the $Q$ maps $\mathcal{J}_{\alpha}(\bar{D})$ into itself. The following estimates hold:

$$
\begin{gathered}
\|W\|_{\alpha, D} \leq\|\Phi\|+\left\|T_{D}(F+G)\right\|_{\alpha, D}, \\
\|H\|_{\alpha, D} \leq\left\|\Phi^{\prime}\right\|+\left\|\prod_{D}(F+G)\right\|_{\alpha, D} .
\end{gathered}
$$

In order to be able to apply the Banach fixed point theorem, we now compare the distance between two elements $(w, h),(\tilde{w}, \tilde{h}) \in \mathcal{J}_{\alpha}(\bar{D})$ and that between their corresponding image $(W, H),(\tilde{W}, \tilde{H})$ under the mapping $Q$. Thus,

$$
\begin{gathered}
\left\{\begin{array}{c}
W=\Phi+T_{D}[F(z, w, h)+G(z, w, \bar{w})] \\
\tilde{W}=\Phi+T_{D}[F(z, \tilde{w}, \tilde{h})+G(z, w, \overline{\tilde{w}})]
\end{array},\right. \\
\left\{\begin{array}{c}
H=\Phi^{\prime}+\prod_{D}[F(z, w, h)+G(z, w, \bar{w})] \\
\tilde{H}=\Phi^{\prime}+\prod_{D}[F(z, \tilde{w}, \tilde{h})+G(z, w, \overline{\tilde{w}})]
\end{array} .\right.
\end{gathered}
$$

It is an immediate consequence that

$$
\begin{gathered}
\|W-\tilde{W}\|_{\alpha} \leq\left\|T_{D}\right\|_{\alpha}\|[F(z, w, h)+G(z, w, \bar{w})]-[F(z, \tilde{w}, \tilde{h})+G(z, w, \overline{\tilde{w}})]\|_{\alpha} \\
\leq L_{1} K_{1}(\alpha, D)\|(w, h)-(\tilde{w}, \tilde{h})\|_{\alpha}+L_{2} K_{2}(\alpha, D)\|w-\tilde{w}\|_{\alpha, D},
\end{gathered}
$$




$$
\begin{gathered}
\|H-\tilde{H}\|_{\alpha} \leq\left\|\prod_{D}\right\|_{\alpha} \|[F(z, w, h)+G(z, w, \bar{w})]-\left[F(z, \tilde{w}, \tilde{h})+G(z, w, \overline{\tilde{w}}) \|_{\alpha}\right. \\
\leq L_{1} K_{3}(\alpha, D) L\|(w, h)-(\tilde{w}, \tilde{h})\|_{\alpha}+L_{2} K_{4}(\alpha, D)\|w-\tilde{w}\|_{\alpha, D},
\end{gathered}
$$

where the constants $K_{1}, K_{2}, K_{3}$ and $K_{4}$ are depending to $\alpha$ and $D$. Then

$$
\|(W, H)-(\tilde{W}, \tilde{H})\|_{\alpha} \leq \max \left(L_{1} K_{1}+L_{2} K_{2}, L_{1} K_{3}+L_{2} K_{4}\right)\|(w, h)-(\tilde{w}, \tilde{h})\|_{\alpha} .
$$

If

$$
0<\max \left(L_{1} K_{1}+L_{2} K_{2}, L_{1} K_{3}+L_{2} K_{4}\right)<1,
$$

then the $Q$ is contractive in $\mathcal{J}_{\alpha}(\bar{D})$. Consequently by fixed point theorem $Q$ has exactly one fixed element $(w, h)$, with the condition $Q(w, h)=(w, h)$ so that

$$
w=\Phi+T_{D}[F(z, w, h)+G(z, w, \bar{w})], \quad h=\Phi^{\prime}+\prod_{D}[F(z, w, h)+G(z, w, \bar{w})] .
$$

By Theorem 1.1, the corresponding $w$ is then a general solution of the partial complex differential equation (1). We now consider the modified RiemannHilbert boundary value problem. We determine the solution $w$ satisfying the following conditions:

$$
\begin{gathered}
\frac{\partial w}{\partial \bar{z}}=F\left(z, w, \frac{\partial w}{\partial z}\right)+G(z, w, \bar{w}), \quad z \in D, \\
\operatorname{Re}(a+i b) w=g \quad \text { on } \quad \partial D,
\end{gathered}
$$

where $a, b, \varphi$ and $g$ are given Hölder continuously differentiable real-valued function of a real parameter $t$ on $\partial D$, and we shall assume that $a^{2}+b^{2}=1$ everywhere on $\partial D$.

It was shown earlier that a general solution $w$ of the partial differential equation (1) has the form

$$
w=\Phi+T_{D}\left[F\left(z, w, \frac{\partial w}{\partial z}\right)+G(z, w, \bar{w})\right]
$$

where $\Phi$ is any function in $C_{1, \alpha}(\bar{D}), 0<\alpha<1$, and holomorphic in $D$. We replace $\Phi$ in (9) by a sum of two holomorphic functions $\phi_{g}$ and $\phi_{(w, h)}$. It follows then from (7) and (8) that

$$
\operatorname{Re}(a+i b)\left[\phi_{g}+\phi_{(w, h)}+T_{D}(F+G)\right]=g,
$$

or

$$
\operatorname{Re}(a+i b) \phi_{g}+\operatorname{Re}(a+i b) \phi_{(w, h)}=g-\operatorname{Re}(a+i b) T_{D}(F+G) .
$$


Thus the modified Riemann-Hilbert problem for $w$ reduces to a similar problem for the holomorphic functions $\phi_{g}$ and $\phi_{(w, h)}$. That is, these functions should satisfy the following conditions:

I. $\operatorname{Re}(a+i b) \phi_{g}=g$,

II. $\operatorname{Re}(a+i b) \phi_{(w, h)}=-\operatorname{Re}(a+i b) T_{D}[F(z, w, h)+G(z, w, \bar{w})]$.

Since both $g$ and $-\operatorname{Re}(a+i b) T_{D}[F(z, w, h)+G(z, w, \bar{w})]$ are in $C_{1, \alpha}(\partial D)$, both problems have a unique solution in $C_{1, \alpha}(\bar{D}),[1]$.

\section{Conclusion}

In this paper we have discussed the modified Riemann-Hilbert boundary value problem for complex partial differential equation (1) and (2) in $C_{1, \alpha}$. We can discuss on the existence and uniqueness of the boundary value problem in the Sobolev space.

\section{References}

[1] A. Seif Mshimba, W. Tutschke, Functional Analytic Methods in Complex Analysis and Applications to partial Differential Equations, ICTP, Trieste (1988), 10-89.

[2] A. Mamourian, Boundary value problems and general systems of nonlinear equations elliptic in the sense of Lavrentiev, Demonstr. Math., XVII, No 3 (1984), 633-645.

[3] A. Mamourian, N. Taghizadeh, Generalization of a first order nonlinear complex elliptic systems of partial differential equations in Sobolev space, Honam. Math. J., 24, No 1 (2002), 67-66.

[4] I.N. Vekua, Generalized Analytic Functions, Pergamon Press, Oxford (1962).

[5] E. Lanckau, W. Tutschke, Complex Analysis Methods Trends and Applications, Pergamon Press, London (1985). 